\title{
Giuseppe Nappi
}

\section{Introduction to the new International Classification of Headache Disorders}

Published online: 20 July 2005

\begin{abstract}
Based on the first edition of the classification (already validated), the ICHD-II is intended for not only the researcher and clinician but also the neurologist and general pactitioner. All the
\end{abstract}

\section{G. Nappi (凶)}

Department of Neurology and Otorhinolaryngology,

University of Rome "La Sapienza",

Rome, Italy and

University Centre for Headache and

Adaptive Disorders (UCADH),

IRCCS Foundation,

Neurological Institute "C. Mondino",

Pavia, Italy

e-mail: giuseppe.nappi@mondino.it

Tel.: +39-0382-380202

Fax: +39-0382-380448

Key words Migraine • Nosography • Primary $\cdot$ Secondary

The main body of the new International Classification of Headache Disorders (ICHD-II) is composed of 4 parts (Table 1). Part I: The primary headaches (migraine, tension-type headache, cluster headache and other trigeminal autonomic cephalalgias and other primary headaches); Part II: The secondary headaches (Chapters 5-12); Part III: The cranial neuralgias and central or primary facial pain and other headaches (Chapters 13 and 14) and Appendix.

The ICHD-II, like the first version, was rigidly structured as a descriptive classification for primary headache disorders, essentially constituting a definition of attacks, with scarce consideration for the evolution of the disease over time. Diagnostic criteria denominated "explicit" were given to each recognised entity. The term "explicit" means unambiguous and precise, based almost exclusively on the clinical characteristics of each single attack, with the scope of leaving as little room as possible for interpretation of the terminology and the symptoms. The classification criterion adopted for secondary headaches is, instead, purely aetiological, even though the structure of the ICHD-II criteria has been standardised and the clinical characteristics of the various forms were added, where available.

Relying on the hierarchical classification already adopted in the first edition, and subsequently validated, the ICHD-II is intended for not only the researcher and clinician but also the neurologist and general practitioner. 
Table 1 Structure of the International Classification of Headache Disorders - II Edition

Part one: The primary headaches

1. Migraine

2. Tension-type headache

3. Cluster headache and other trigeminal autonomic cephalalgias

4. Other primary headaches

Part two: The secondary headaches

5. Headache attributed to head and/or neck trauma

6. Headache attributed to cranial or cervical vascular disorder

7. Headache attributed to non-vascular intracranial disorder

8. Headache attributed to a substance or its withdrawal

9. Headache attributed to infection

10. Headache attributed to disorder of homeostasis

11. Headache or facial pain attributed to disorder of cranium, neck, eyes, ears, nose, sinuses, teeth, mouth or other facial or cranial structures

12. Headache attributed to psychiatric disorder

Part three: Cranial neuralgias, central or primary facial pain and other headaches

13. Cranial neuralgias and central causes of facial pain

14. Other headache, cranial neuralgia, central or primary facial pain

Appendix

All the headaches are classified into groups, which are then subdivided one, two or three times to provide the level of diagnosis necessary for each user.

The scarce attention given by the ICHD-II to the evolution of headaches could represent a limitation of the taxonomical system in clinical practice; nevertheless, this position was the result of an attentive and well-pondered choice, suggested by the lack of reliable information on the evolution of numerous forms of headaches, especially the primary types. Another aspect that has had a scarce impact on the classification is the one related to genetic findings: despite the volume of data accumulated over the years, to the present day it has not been possible to identify monogenetic forms within the heterogenous group of phenotypes clinically described.

This was done with the purpose of stimulating the collection of evidence-based information that will eventually lead to the correct nosography. The ICHD-II Committee has created the Appendix, a section in which the headache forms that have not been clinically and diagnostically classified or diagnostic criteria that must be validated by research studies have been temporarily included.

\section{Suggested readings}

Headache Classification Subcommittee of the International Headache Society (2004) The International Classification of Headache Disorders, 2nd edn. Cephalalgia 24[Suppl 1]:9-160
Nappi G, Manzoni GC (2005) Manuale delle Cefalee. Masson, Milano
Nappi G, Rossi P (2004) Migraine - current concepts. Current Medical Literature, London 